\title{
Kapitel 7 - Fazit der Arbeit
}

Der Ausgangspunkt der vorliegenden Arbeit lag in der Feststellung, dass mit aktuellen antisemitischen Ereignissen oftmals nicht in der Art und Weise umgegangen wird, wie es aus antisemitismuskritischer und demokratischer Perspektive wünschenswert wäre. Problematisch ist insbesondere, dass die Existenz von Antisemitismus in seinen verschiedenen Formen oftmals nicht anerkannt und kritisiert und die Kritik antisemitischer Menschen aktiv behindert wird. Die Frage, wie solche Umgangsweisen mit Antisemitismus und wie solche Reaktionen auf Antisemitismuskritik zu beschreiben sind, mit welchen Motiven sie verknüpft und mit welchem theoretischen Instrumentarium sie $\mathrm{zu}$ untersuchen sind, verweist auf den Kerngegenstand der vorliegenden Studie.

In diesem die Arbeit abschließenden Kapitel werden die Ergebnisse nun zusammengefasst und der Bogen von den eingangs gestellten Forschungsfragen über die theoretischen Überlegungen und Konzepte zu den empirischen Ergebnissen gespannt. In den Kapiteln 7.1 und 7.2 werden die Forschungsfragen beantwortet werden. Dies geschieht zunächst begrifflich im Fazit zur Theoriebildung (7.1) und dann materialgesättigt im Fazit zur Empirie (7.2). In Kapitel 7.3 werden dann einige Begrenztheiten der Studie benannt und die Geltungsreichweite der gewonnenen Ergebnisse diskutiert. In 7.4 wird auf empirische, theoretische und praktische Anschlussfragen eingegangen, die sich in Anschluss an die Befunde der Arbeit ergeben und auf mögliche zukünftige Forschungsprojekte verwiesen. Die Arbeit schließt mit Kapitel 7.5.

\subsection{Fazit der Theoriebildung}

Für die Modellierung eines theoretischen Ansatzes, mit dem typische Reaktionen auf anti-antisemitische Interventionen sowohl beschrieben als auch er- 
klärt werden können, habe ich vorgeschlagen, den psychoanalytisch geprägten Begriff der Abwehr aufzunehmen und unter Berücksichtigung soziologischer Erkenntnisse systematisch zu erweitern. Notwendig war diese Modellierung, um die ganze Bandbreite von im qualitativen Material beobachtbaren Verhaltensweisen im Rahmen eines einheitlichen Modells beschreiben und erklären zu können.

Die metapsychologische Konzeptualisierung der Abwehr erweist sich in diesem Zusammenhang als Vorbild für die sozialwissenschaftliche. Als brückentheoretische Annahme dient hierbei insbesondere die Überlegung, dass psychische und soziale Abwehrvollzüge gemein haben, dass sie als mehr oder weniger auffällige Formen der funktionalen Unterbrechung von Kommunikation betrachtet werden sollten. Das ideelle Verwandtschaftsverhältnis scheint hierbei selbst noch in der von der Psychoanalyse scheinbar am weitesten entfernten systemtheoretischen Konzeptualisierung auf, wo Latenz als funktionale Kommunikationsblockade definiert wird. Es zeigt sich damit, dass der Versuch, Abwehr soziologisch zu fassen, kein Novum darstellt. Meines Erachtens neu ist allerdings der Versuch die Zusammenhänge zwischen verschiedenen theoretischen Ansätzen - d.h. psychoanalytischen, sprechakttheoretischen und systemtheoretischen - aufzuzeigen und vor diesem Hintergrund einen Katalog sozialer Abwehrhandlungen in systematisierender Weise darzustellen und auf das Feld des Umgangs mit Antisemitismus und Antisemitismuskritik anzuwenden.

Die Abgrenzung der soziologischen von der psychoanalytischen Begriffsbildung erfolgt insbesondere hinsichtlich der Bestimmung der Lokalität, an der Kommunikation abgebrochen wird. Während die Psychoanalyse betont, dass es sich bei den Abwehrmechanismen um »Vorgänge [handelt], die sich im Individuum selbst abspielen und sich auch ausschließlich auf das Individualpsychologische beschränken« (Mentzos 1976: 10), so handelt es sich bei sozialen Abwehrhandlungen um Vorgänge, die nicht im Individuum, sondern gewissermaßen zwischen Individuen situiert sind. D.h., Abwehrhandlungen spielen nicht in intra-individuellen, sondern in interpersonalen Konflikten eine Rolle. Abwehrhandlungen zielen hierbei auf die Abmilderung oder sogar Umkehrung der (tatsächlichen, möglichen oder imaginierten) Wirkungen, die von anti-antisemitischen Interventionen bzw. der Erinnerung an den Antisemitismus relevanter anderer ausgehen. Diese Definition führt dazu, dass die Vorstellung aufgeben muss, dass Abwehrhandlungen immer auf einen intra-individuellen Konflikt, z.B. latente Schuldgefühle, zurückgeführt werden können. Als soziale Handlungen gedacht muss zwar die (latente) Wahrneh- 
mung eines zu neutralisierenden Konflikts vorausgesetzt werden, dieser ist allerdings nicht primär ein interner, sondern ein (potenzieller, latenter oder manifester) Konflikt zwischen mehreren Personen.

Aus dieser Konzeptualisierung lassen sich verschiedene Schlussfolgerungen ziehen. So sollte z.B. von einer allzu voreiligen Vermengung des metapsychologischen Konzepts der Abwehrmechanismen mit dem soziologisch beschreibbaren Konzept der Abwehrhandlungen abgesehen werden. Eine Pointe des metapsychologischen Begriffs besteht darin, davon auszugehen, dass innerpsychische Konflikte durch Formen der Abwehrargumentation zugleich zum Ausdruck gebracht wie auch verborgen werden. Dementsprechend läge es dieser Perspektive nahe, z.B. Formen der moralischen Diskreditierung von Kritikern als »Projektion« abgespaltener Aggressionen, die Externalisierung von Antisemitismus als »Abspaltung« verdrängter antisemitischer Einstellungen zu interpretieren. Demgegenüber besteht ein Kerngedanke des hier entwickelten soziologischen Begriffs der Abwehr darin, dieses als Handeln zu bestimmen, das soziale Wirkungen entfalten soll. Demnach wird durch Abwehrhandlungen als soziale Handlung nicht (oder zumindest nicht primär) angestrebt, auch etwas psychologisch Verdrängtes zur Sprache zu bringen, sondern es steht der gewünschte Effekt der Neutralisierung von Sozialbeziehungen gefährdenden, anti-antisemitischen Interventionen in sozial akzeptabler Form im Vordergrund. ${ }^{1}$

Hinsichtlich der Frage, auf welche Konflikte sich abwehrendes Handeln bezieht, sollte heuristisch zwischen zwei Fällen unterschieden werden. Im ersten Fall zielen Abwehrhandlungen darauf, einen latenten Konflikt latent $z u$ halten. Im zweiten zielen solche Handlungen darauf, einen manifesten Konflikt wieder in die Latenz zu überführen. Abwehrendes Verhalten ist demnach in manchen Fällen als Reaktion auf tatsächliche Kritik oder die Erinnerung an Antisemitismus zu betrachten, in anderen Fällen ist sie als proaktives, d.h. vorausplanendes und kritische Interventionen antizipierendes Handeln zu verstehen. Je nach Konfliktlage und Interesse unterscheiden sich hierbei auch die Strategien, mit denen dies erreicht werden kann. Diesbezüglich konnte in Kapitel 2 gezeigt werden, dass Individuen in ihrem Bemühen, antisemitische Konflikte zu neutralisieren, eine große Palette von konkreten Handlungen zur Verfügung stehen: so z.B. das Vermeiden von Themen oder der Handlungen auf die Erweiterung des Sagbaren, bringen sie zwar nicht psychologisch, aber kommunikativ Verdrängtes in den Diskurs ein. 
Mitteilung persönlicher Einstellungen, ein Schweigen an der richtigen Stelle im gemeinsamen Gespräch, ebenso wie ein abrupter Themenwechsel oder sogar das vermeintlich mitteilsame Sprechen. Abwehr umfasst so gegensätzliche Handlungen wie die Leugnung, Bagatellisierung und explizite Rechtfertigung von Antisemitismus oder die Diskreditierung der Kritiker*innen von Antisemitismus. Die Frage, welche spezifische Strategie zum Einsatz kommt, hängt hierbei auch mit den Spezifika des sozialen Konflikts zusammen. Es ist hierbei die Aufgabe der deutenden Sozialforschung, Annahmen darüber anzustellen, auf welche Konfliktkonstellation ein bestimmtes Handeln überhaupt bezogen ist.

Ein weiterer wichtiger Befund bestand in der Feststellung des in zweierlei Sinn überindividuellen Charakters von Abwehrhandlungen. Erstens bleibt festzuhalten, dass soziale Abwehrhandlungen - insbesondere Abwehrargumentationen - nicht erst individuell erfunden werden müssen, sondern Individuen aus einem ganzen Fundus von stereotypen, gesellschaftlich tradierten Abwehrhandlungen auswählen können und sich in ihrer Aktualisierung wiederum soziale Muster bilden. Zweites legen die theoretischen Befunde nahe, dass es zu einer lebensweltlichen Verankerung von Überzeugungen kommt, die dem Interesse der Antisemitismuskritik, Antisemitismus sicht- und angreifbar zu machen, diametral gegenüberstehen, wenn sich bestimmte Typen abwehrenden Handelns wiederholt als erfolgreich erweisen. Diese Feststellung führt allerdings auch zu einer Problematik, die mit der Anwendung dieses Theorieansatzes verbunden ist. Denn zweifelsohne ist der Unterschied zwischen Antisemitismus dethematisierenden Argumentationen, die einen funktionalen Charakter haben und solchen, die aus wertrationalen Erwägungen heraus formuliert werden, zwar theoretisch zu plausibilisieren. In der Analyse von empirischen Beispielen ist es bisweilen allerdings nicht einfach, eine entsprechende Unterscheidung zu treffen. Die Ursachen hierfür liegen in der Sache selbst. Denn wenn Abwehrhandlungen darauf abzielen, die ihnen zugrundeliegenden Motive zu verschleiern, d.h. z.B. unter instrumentellem Bezug auf sozial akzeptierte bzw. akzeptierbare Normen und Situationsbeschreibungen, dann muss auch die Kritik dieser Handlungen notgedrungen sinvestigativ vorgehen. Unter Rückgriff auf Kontextinformationen müssen Hypothesen zu entsprechenden Motiven aufgestellt oder es müssen Widersprüche und Inkonsistenzen des Materials bzw. des Verhaltens einzelner in verschiedenen Situationen herausgearbeitet werden. Diese dürfen sodann nicht als Ausdruck der Inkonsistenz menschlichen Handelns überhaupt bzw. als Ausdruck von individuellen Ambivalenzen, sondern als Hinweis auf 
latente Motive interpretiert werden, die dieses Verhalten als zweckrationales ausweisen. Deshalb sei betont, dass die in der Studie vorgenommenen Deutungen nur als Angebote verstanden werden können, auch weil in den empirischen Analysen schon allein der Darstellung wegen nicht alle Möglichkeiten für die Erklärung je spezifischen Handelns ausgeschöpft wurden. Ein solches Vorgehen hätte den Umfang der Fallstudien gesprengt. Dies gilt u.a. auch hinsichtlich der in verschiedenen Teilkapiteln vorgenommenen Bezüge auf den Hostile Media Effect. In Kapitel 1.1 wurden damit verbundene theoretische Annahmen in Zusammenhang mit dem Begriff der Abwehr in der Psychoanalyse gestellt. Hierzu sollte ergänzt werden, dass die Annahme, dass Menschen durch die Stilisierung von Medien zum Feind eine kognitive Dissonanz bewältigen, in der Theoriediskussion zu diesem Phänomen eher die Ausnahme als die Regel darstellt und andere Erklärungsversuche vorherrschen (vgl. Huge/Glynn 2010: 166f., Feldman 2017).

Freilich hätte eine andere Möglichkeit der Theoriebildung auch darin bestehen können, Abwehrhandlungen weniger über damit verbundene verdeckte Motive, sondern ausschließlich über ihren Effekt - die Neutralisierung eines aus Beobachterperspektive existierenden Konfliktes - zu bestimmen. Allerdings wäre dies m.E. mit der Gefahr eines undifferenzierten Ausuferns des Begriffs verbunden. Die Kritik Anja Weiß' an entsprechenden Ansätzen der Rassismusforschung, die das Phänomen auf rassistische Effekte zeitigende Handlungen reduzieren, lässt sich hierbei auch auf den Gegenstand der Abwehrhandlung übertragen (vgl. Weiß 2001: 39f.). ${ }^{2}$ Von einer solchen Verkürzung grenzt sich sie die vorliegende Arbeit $\mathrm{ab}$, indem sie die Identität der drei Ebenen des Abwehrbegriffs (Funktionszusammenhänge - Verhaltensmuster - Effektmuster) betont.

Alternativ hätte ich auch, bei Bergmann und Erb ansetzend, die Funktion von Abwehrhandlungen im sozialen oder politischen System verorten können, ohne sie im Sinne eines methodologischen Individualismus als subjektiv funktional auszuweisen. In diesem Fall hätte die vorliegende Arbeit einen Fokus auf die latente Funktionalität der Abwehr für soziale und politische Institutionen und Gruppen zum Gegenstand gehabt. Dieser stärker gruppensoziologisch und politikwissenschaftlich orientierte Forschungsfokus könnte eines antisemitischen Konfliktes führen. Es wäre allerdings unsinnig, sie (pauschal) zur Abwehrhandlung zu erklären. 
sich für zukünftige Studien als produktiv erweisen und müsste, im Gegensatz zur vorliegenden Studie, Beobachtungen auf der gesellschaftlichen Makround Meso-Ebene vornehmen.

\subsection{Fazit der empirischen Untersuchung}

Während im vorangegangenen Kapitel bestimmte Umgangsweisen mit Antisemitismus bzw. dessen Thematisierung und Kritik in begrifflicher Hinsicht unter dem Begriff der Abwehr gefasst wurden, folgt nun eine Beantwortung der drei Forschungsfragen in empirischer Hinsicht. So wurde gefragt,

- wie auf die Kommunikation von Antisemitismus und wie auf dessen potenzielle oder tatsächliche Kritik reagiert wird,

- wie sich diese Verhaltensweisen erklären lassen,

- warum in Debatten über geäußerten Antisemitismus Sympathien häufig für diejenigen empfunden werden, die Antisemitismus äußern.

Um diese Forschungsfragen zu beantworten, wurde vorgeschlagen, zwei verschiedene Formen qualitativer Methoden - die fokussierte Gruppendiskussion und das fokussierte Interview - miteinander zu verbinden. Während sich durch die Gruppendiskussionen die spontanen Reaktionen von Teilnehmer*innen auf einen antisemitischen Konflikt in einer offenen Gesprächssituation erheben ließen, diente das Material der Interviews dazu, an Daten zu gelangen, mit denen die individuellen Bedeutungen entsprechender Reaktionen erschlossen werden konnten. Es wurden die Reaktionsweisen von insgesamt dreiundzwanzig Studierenden auf die Problematisierung von israelbezogenem Antisemitismus in der 2012 stattgefundenen Debatte über das Gedicht des Nobelpreisträgers Günter Grass »Was gesagt werden muss « ${ }^{3}$ und hieran anschließende Diskurse und Äußerungen untersucht.

3 Während die Beurteilung von Grass' Beitrag zur deutschen Nachkriegsliteratur und sein Bemühen und Beitrag zur Aufarbeitung der deutschen Vergangenheit nicht Cegenstand der vorliegenden Arbeit waren, so nahm sie insgesamt eine unmissverständlich kritische Haltung zum Grass'schen Gedicht ein, dessen Inhalte sie als nicht rechtfertigbar und als Reproduktion antisemitischer Vorurteile und Stereotype beurteilt. 
Folgende Liste gibt einen verdichteten Überblick über wiederkehrende Reaktionen auf antisemitische Äußerungen sowie auf die potenzielle oder tatsächliche Kritik antisemitischer Ereignisse:

- Ausbleibender Widerspruch gegen die antisemitischen Äußerungen im Grass-Gedicht sowie hinsichtlich von artikuliertem und beobachtetem Antisemitismus im sozialen und politischen Umfeld der Teilnehmer*innen

- Positive Umdeutungen antisemitischer Äußerungen (von Grass und sozial und politisch nahestehenden Personen und Gruppen)

- Unverständnis gegenüber und teilweise mit antisemitischen Stereotypen verbundene Kritik an anti-antisemitischen Interventionen

- Sprachliches Latent-Halten der Shoah durch die Verwendung von Leerwörtern und Euphemismen

- Kontrafaktische Behauptung eines Verbots, Juden oder Israel in Deutschland kritisieren zu dürfen

- Stereotype und auf antisemitische Stereotypen bezugnehmende Negativdarstellungen Israels

- Darstellungen von Antisemitismus als Problem anderer Zeiten und Gruppen

- Beschreibungen von Antisemitismus, die ihn extremisieren oder bagatellisieren

- Erklärungen von Antisemitismus, die >Antisemiten lächerlich machen oder Antisemitismus auf das Handeln von Jüd*innen zurückführen

- Vereinzelt: Jüd*innen herabwürdigende oder offen antidemokratische Äußerungen

Zur Erklärung dieser Verhaltensweisen wurden - je nach Kontext und Fall verschiedene Hypothesen formuliert, wobei an die Befunde der hier dargelegten Theorie angeschlossen werden konnte. Dies führte in verschiedenen Variationen zu Hypothesen, die sich in der nun folgenden Verdichtung zusammenfassen lassen. Die Zurückweisung von anti-antisemitischen Interventionen und die Dethematisierung von Rassismus und Antisemitismus sowie die Nicht-Thematisierung nationalistischer und rassismus- oder antisemitismusrelevanter Einstellungen scheint demnach im untersuchten Material die Funktionen zu erfüllen, 
- eine Normalisierung deutsch-jüdischer Beziehungen, des deutsch-deutschen Selbstverhältnisses oder einen neuen Nationalstolz überhaupt erst einfordern zu können, ohne sich als revisionistisch darstellen zu müssen,

- das identitätsstiftende Konstrukt einer moralischen deutschen Gemeinschaft gegen Infragestellung abzuschirmen,

- die Beziehung zu relevanten anderen (d.h. Großeltern, politisch Nahestehenden, anderen Teilnehmer*innen der Gruppendiskussion bzw. dem Interviewer) zu stabilisieren,

- den sozialen Druck auf eigene antidemokratische bzw. (israelbezogene) antisemitische Einstellungen zu senken bzw. die Grenzen des Sagbaren so zu verschieben, dass diese Einstellungen zukünftig sagbar werden könnten.

Allerdings wurde auch darauf hingewiesen, dass es verkürzt sein könnte, alle Reaktionen auf das Vorliegen solcher latenten Motive (und damit letztlich auf ein zweckorientiertes Handeln) zurückzuführen. Dies kann hinsichtlich der Grass-Debatte noch einmal exemplarisch an drei Kommunikationsmustern verdeutlicht werden.

\section{Verbreitete Israelbilder}

In Kapitel 5.3 wurde gezeigt, dass viele Teilnehmer*innen Israel oder Israelis zum wesentlichen Problemakteur im Nahen Osten stilisieren. Israel sowie Israelis werden als eine Gruppe wahrgenommen, die aggressiv, gewaltbereit sowie unzurechnungsfähig und irrational sei und durch das eigene Verhalten Gegenwehr provoziere. Hinter der Forderung nach Israelkritik verbergen sich demnach häufig Formen einseitiger und mitunter antisemitischer Darstellungen, deren Legitimität eingefordert wird. Zudem wurde deutlich, dass dies häufig mit einer nicht-Thematisierung der Politik des Irans einhergeht. In Anschluss an die Theorie wurden diese Darstellungen als Reproduktion der unter 2.3 diskutierten Rechtfertigungen von Antisemitismus interpretiert.

Auf der anderen Seite könnten solche Israelbilder auch Ausdruck eines, z.B. durch Medienkonsum oder alltägliche Kommunikation angeeigneten, "Wissens« interpretiert werden. So hat etwa Robert Beyer die These aufgestellt, dass die Einstellung der Bevölkerung durch Negativdarstellungen Israels nachhaltig beeinflusst wird (vgl. Beyer 2015: 219). In diesem Fall müsste die Existenz dieser negativen und teils antisemitischen Israelbilder als Ausdruck gesellschaftlich geformter Überzeugungen gelesen werden, die 
keine unmittelbaren Funktionen erfüllen. Die Zurückweisung von anti-antisemitischen Interventionen wäre demnach als Ausdruck der Überzeugung $\mathrm{zu}$ verstehen, dass weil Israel ein Problemakteur sei, es sich beim GrassGedicht um eine sinnvolle >Israelkritik handeln müsse. ${ }^{4}$ Die Zurückweisung von Kritik müsste dann nicht als Abwehrhandeln, sondern als Ausdruck wertrationalen Handelns verstanden werden. ${ }^{5}$

\section{Behauptung von Israelkritik als Tabu}

Es wurde gezeigt, dass etliche Teilnehmer*innen behaupteten, dass eine Kritik israelischer Politik in Deutschland generell verboten und sanktioniert sei. Mehrere in diesem Zusammenhang vorgenommenen Analysen zeigten hierbei, dass einige Teilnehmer*innen beim Versuch die Behauptung zu plausibilisieren, wiederholt Geschichten über entsprechende Tabuisierungen serfinden mussten. Entsprechende kontrafaktische Konstruktionen (d.h. insbesondere die Dramatisierung der Situation und die Verwendung von Strohmännern und sekundärantisemitischen Motiven) ließen sich hierbei auch in Reaktionen auf das Stimulusmaterial identifizieren. Hier reagierten Teilnehmer*innen auf die anti-antisemitischen Interventionen mit Unverständnis, welches aber durch die eigenen, verzerrenden Interpretationen des Ausgangsmaterials selbst bedingt war. Dies kann als Hinweis darauf gelesen werden, dass diese negativen Reaktionen nicht als Resultat der Erfahrungen mer*innen, die sich mit Grass solidarisieren, als wesentlicher Problemakteur im Nahen Osten, als irrational, aggressiv, gefährlich oder sogar gemeingefährlich dargestellt. Sechs dieser Teilnehmer*innen äußerte sich zudem auf antisemitische oder sekundärantisemitische Weise. Umgekehrt gibt es allerdings auch fünf Personen, die sich mit Grass solidarisieren, aber keine antiisraelischen oder antisemitischen Einstellungen zum Ausdruck bringen. D.h. auch Menschen, bei denen ein anti-israelischer Bias nicht vorzuliegen scheint, reagieren mit Ablehnung auf den ZDF-Bericht. In diesem Zusammenhang ist zusätzlich darauf hinzuweisen, dass es aufgrund der Datenlage nicht möglich war, zu untersuchen, wie sich die Existenz dieser Wahrnehmung Israels verstehend erklären lässt. So könnte zum einen die Hypothese aufgestellt werden, dass diese Israelbilder für die Teilnehmer*innen auch psychologische Funktionen, z.B. im Sinne einer Schuldnivellierung, erfüllen. Würde dies zutreffen, so könnte gefolgert werden, dass die Zurückweisung von anti-antisemitischen Interventionen auch die Funktion erfüllt, solche individuell funktionalen Bilder gegen ihre Kritik abzuschirmen. Weder das untersuchte Material noch die gewählte Auswertungsstrategie lassen solche tiefenhermeneutischen Schlüsse allerdings zu. 
mit willkürlichen oder instrumentalisierten Antisemitismusvorwürfen (und damit als wertrationale Reaktionen) verstanden werden sollten, sondern als Ausdruck von Abwehrverhalten interpretiert werden können, die den Zweck erfüllen Gegenstimmen zu delegitimieren.

Auf der anderen Seite könnten solche Darstellungen in manchen Fällen auch als Resultat einer aus gesellschaftlichen Gerüchtebildungsprozessen resultierende Überzeugung interpretiert werden. Dementsprechend könnte die Rede vom »Kritiktabu « auch als lebensweltlich verbreitetes Deutungsmuster bezeichnet werden, dessen Für-Wahr-Halten die Ablehnung von anti-antisemitischen Interventionen bedingt. Denn wird die populäre These eines Kritikverbots für wahr gehalten, so liegt es nahe zu schlussfolgern, dass es auch im Falle des Grass-Gedichts zu unfairen Anklagen kommt.

\section{Reduktionistische Vorstellungen von Antisemitismus}

Die im Material untersuchten Laientheorien zu Antisemitismus haben gezeigt, dass die Teilnehmer*innen zu großen Teilen Antisemitismuskonzepte vertreten, die vor dem Hintergrund der aktuellen Forschung als antiquiert, im Falle korrespondenztheoretischer Annahmen sogar als äußerst problematisch bewertet werden müssen, insofern hierbei der Antisemitismus direkt oder indirekt auf das Verhalten von Jüd*innen zurückgeführt wird. Diese Überzeugungen können auf zweierlei Weise gedeutet werden. Demnach kommt solchen Überzeugungen entweder die Funktion zu, Abwehrargumentationen zu plausibilisieren. D.h., wenn Antisemitismus als exzeptionelles oder extremes Phänomen präsentiert werden kann, so sinkt die Chance, dass der »alltägliche« Antisemitismus als Gewaltproblematik erscheinen kann. Alternativ hierzu kann in Anschluss an die Soziologie sozialer Probleme allerdings auch davon ausgegangen werden, dass ein »bestimmter Sachverhalt weniger aufgrund objektiver Faktoren, sondern vielmehr aufgrund von gesellschaftlichen Definitionsleistungen und diskursiven Prozessen als >Problem $<$ wahrgenommen wird - oder eben nicht.« (vgl. Arnold: 2016: 410). Das bedeutet, wenn sich die im Material dokumentierten, reduktionistischen Ansichten zu Antisemitismus als Ausdruck eines gesellschaftlich tradierten Wissen erweisen, dann ist es nicht überraschend, dass aktueller Antisemitismus nicht erkannt wird. ${ }^{6}$ In diesem Fall wäre auch der These von Betzler/Glittenberg zuzustim- 
men, dass die berechtigte Kritik von israelbezogenem Antisemitismus für illegitime Antisemitismusvorwürfe gehalten werden, wenn der israelbezogene Antisemitismus nicht erkannt wird: »Auf Grundlage einer vielfachen Wahrnehmung dieser Dynamik ist dann von einer >Inflationierung des Begriffs Antisemit(ismus)< die Rede« (Betzler/Glittenberg 2015: 277).

\section{Gründe für Sympathien mit Grass}

Abschließend soll hier die dritte Forschungsfrage beantwortet werden, warum die verschiedenen Teilnehmer*innen Sympathien für den Autor Grass empfinden. Ein interessantes Ergebnis war hierbei, dass bei vielen der Teilnehmer*innen Sympathien mit Grass wenig oder nur unter anderem mit ihren Wahrnehmungen Israels, des Nahostkonflikts und dem israelisch-iranischen Konflikts zu tun hatten. Vielmehr legen die Analysen nahe, dass es lebensweltlich bedeutsame und konfliktbeladene Thematiken sowie damit verbundene Lösungs- und Wunschvorstellungen $\mathrm{zu}$ sein scheinen, die auf den Grass-Konflikt »übertragen « werden, obgleich sie diesem Konflikt inhaltlich völlig unangemessen sind. ${ }^{7}$ Sympathien bestehen demnach deshalb, weil

- Grass als Bündnispartner im Kampf gegen die vermeintliche Dominanz oder Veränderungsresistenz problematischer politischer Verhältnisse (insbesondere in Bezug auf Parteipolitik und "politische Korrektheit«) wahrgenommen wird,

- Grass' negative Haltung zu Israel, Israelis, israelischer Politik und >dem (amerikanischen) Westen $`$ geteilt wird,

- Waffenlieferungen im Sinne einer pazifistischen oder nationalistischen Einstellung als Problem bzw. als Risiko wahrgenommen werden,

- die eigene soziale Situation (wegen einer potenziellen oder tatsächlichen Stigmatisierung der eigenen politischen Meinung) der von Grass ähnlich erscheint.

korrespondenztheoretische Erklärungsansätze vertrat, dass er »Mein Kampf« gelesen habe, um zu verstehen, warum die Juden im Nationalsozialismus so gehasst worden seien (vgl. TL-L: 8f.). Hier ist eine Antisemitismus in Facetten legitimierende Argumentation paradoxer Weise auf ein anti-antisemitisches Interesse zurückführbar.

7 Das Phänomen der Übertragung ist nicht als ein für Gespräche über Antisemitismus oder Vorurteile spezifischer, sondern als ein ständig in politischen Alltagsgesprächen mitlaufender Prozess zu betrachten (vgl. Volmerg et al. 1983: 385). 
Hier zeigt sich, dass die Motive Grass zu verteidigen genauso vielfältig sind, wie die oben diskutierten Motive, Antisemitismus zu dethematisieren. Hieraus sollte der Schluss gezogen werden, dass sich die empirische Sozialforschung in der Analyse antisemitischer Konflikte nicht mit einfachen Antworten zufriedengeben sollte, sondern es lohnend ist, sich auf die Komplexität des Gegenstands einzulassen.

\subsection{Grenzen und Geltungsreichweite der Ergebnisse}

Wie bereits in der Einleitung festgestellt wurde, handelt es sich bei der vorliegenden Arbeit um eine Fallstudie, die insbesondere der Exploration eines bestimmten Forschungsfeldes sowie der Theoriegenerierung dient. Aus diesem Charakter der Arbeit ergibt sich zugleich eine Einschränkung der Geltungsreichweite der Befunde aufgrund der untersuchten Personengruppe, der Zahl der untersuchten Personen, der Spezifika des Untersuchungsaufbaus, sowie der Qualität des untersuchten Materials.

\section{Wahl der Personengruppe und der Anzahl der Interviewten}

Bezüglich der untersuchten Personengruppe ist festzuhalten, dass aufgrund des qualitativen Fokus der Arbeit und aufgrund begrenzter Arbeitsressourcen nur dreiundzwanzig Teilnehmer*innen befragt werden konnten. Hierdurch wurde zwar eine große Tiefe des Informationsgehalts des Materials sichergestellt und eine Analyse individueller Motivlagen ermöglicht, allerdings kann damit kein umfassender Anspruch auf Repräsentativität und Relevanz der Ergebnisse erhoben werden. Die Frage, inwiefern die Ergebnisse der vorliegenden Studie hinsichtlich der Gesamtgruppe von Studierenden wirklich verallgemeinerbar sind, könnte durch die Durchführung einer quantitativen Studie (siehe Kapitel 7.4) überprüft werden. Zudem führt die Wahl von Studierenden als untersuchter Gruppe dazu, dass keine systematischen Vergleiche zu anderen Personengruppen möglich sind, die Rückschlüsse auf die Besonderheit von Verhaltensmustern zulassen. Allerdings scheint es mir plausibel davon auszugehen, dass die untersuchte Stichprobe aufgrund der ausgeglichenen Zusammenstellung hinsichtlich der Kategorien Geschlecht, politische Orientierung, Alter und Studienfach in Hinsicht auf der Gesamtgruppe keine groben Verzerrungen aufweist. Es ist also wahrscheinlich, dass sich die Er- 
gebnisse nicht nur auf eine kleine Gruppe speziell interessierter Studierender beziehen lassen.

\section{Keine Variation des Untersuchungsaufbaus}

Eine zweite Einschränkung der Geltungsreichweite der Ergebnisse ergibt sich aus der spezifischen Ausgestaltung des Forschungsdesigns, das auf mehreren Selektionen beruhte. So wurde nicht die Frage nach dem Thema Antisemitismus im Allgemeinen zum Ausgangspunkt der Diskussionen gemacht, sondern ein spezifischer antisemitischer Konflikt und ein spezifisches Konfliktthema (israelbezogener Antisemitismus) gewählt. Auch der Grass-Konflikt wurde nur in einem Ausschnitt präsentiert. Der Vorteil dieses Vorgehens bestand darin, den Untersuchungsgegenstand in seinem natürlichen Umfeld mit großer Tiefenschärfe untersuchbar zu machen. Allerdings stellt sich die Frage, ob sich die gleichen Teilnehmer*innen anders verhalten hätten, wenn der Grundreiz auf differente Weise konzipiert worden wäre, z.B. durch die Auswahl einer anderen Debatte, anderer Medienbeiträge oder anderer kritischer Stimmen zu Günther Grass. Relevant ist diese Frage, weil z.B. aus den Forschungen zu den >Milgram-Experimenten bereits kleine Veränderungen des Untersuchungsaufbaus zu Veränderungen in den beobachtbaren Reaktionen von Untersuchungsteilnehmer*innen führen können (vgl. Milgram 1965). So hätte z.B. das Gedicht nicht mit dem für Gedichte typischen stragenden Verlesungsstil per Video vorgetragen, sondern eine semotionalere Präsentationsvariante gewählt werden können, um zu überprüfen, ob die Reaktionen der Teilnehmer*innen dann stärker variieren. Alternativ hätten auch andere Formen anti-antisemitischer Kommentare verwendet oder einzelne Kommentare weggelassen werden können. Durch solche Variationen hätte z.B. genauer die Frage beantwortet werden können, welche >Trigger Abwehrhandlungen eher auslösen und ob die Zustimmungsbereitschaft $\mathrm{zu}$ anti-antisemitischen Interventionen erhöht werden kann. ${ }^{8}$

8 Eine solche Analyse solcher Trigger in Diskursen über Rassismus hat Robin DiAngelo vorgenommen (vgl. dies. 2001: 57). Sie zählt dazu unter anderem die Problematisierung von Rassismus, die Infragestellung von dominanten Perspektiven auf Rassismus in der Mehrheitsgesellschaft und die Konfrontation mit Persons of Color in relativen Machtpositionen. 
Insgesamt lässt sich bezüglich des trotz seiner Länge immer noch knapp gehaltenen Grundreizes festhalten, dass dieser auf ein allgemeines Problem medial induzierter Meinungsbildungsprozesse verweist. Denn, Jürgen Habermas zufolge, führt »die Knappheit der funktional notwendigen Ressourcen, von denen insbesondere deliberative Meinungs- und Willensbildungsprozesse in hohem Maße abhängen " (Habermas 1992: 396), zu Verzerrungen und Abweichungen vom formulierten Diskursideal. Bezogen auf den ZDFBericht bedeutet dies, dass dessen Art der Vermittlung eines antisemitischen Konflikts (politische Positionierungen ohne Hintergrundinformationen) auch als Teil des in dieser Arbeit diskutierten Problems der Ablehnung anti-antisemitischer Interventionen betrachtet werden kann. Wie bereits oben festgestellt, ist es aufgrund der Nicht-Variation des Untersuchungsaufbaus nicht möglich zu untersuchen, ob es andere Vermittlungsformen antisemitismuskritischer Meinungen (z.B. mit zusätzlichen Sachinformationen) gibt, die einen positiveren Effekt auf Seite der Rezipient*innen hervorrufen.

\section{Künstlicher Kontext der Befragung}

Nicht überflüssig festzustellen ist abschließend, dass sich eine weitere Einschränkung der Geltungsreichweite der Untersuchungsergebnisse aus der Beeinflussung der teilnehmenden Personen durch den artifiziellen Charakter der Untersuchung ergeben hat. Wie deutlich gemacht wurde, erzeugen tatsächliche, vermeintliche oder mögliche anti-antisemitische Interventionen den Bedarf an Abwehrhandlungen, der nicht bestünde, hätte sich nicht eine anti-antisemitische Kultur etabliert, die - wie Abwehrhandlungen auch im Kontext sozialer Situationen aktualisiert wird. Dies bedeutet, dass Abwehrhandlungen ohne die Unterstellung einer anwesenden und potentiell kritischen Öffentlichkeit keinen Sinn ergeben. Abwehrhandlungen vollziehen sich also soziologisch verstanden immer »im Horizont des Publikums« (Werron 2009: 15). $9^{9}$ Dieser Horizont wurde im Rahmen der Datenerhebung nicht allein durch die Wahrnehmung der Interviewsituation durch die Teilnehmenden, sondern auch durch die Wahrnehmung und den Effekt der Äußerungen des interviewenden Wissenschaftlers sowie der anderen, meist unbekannten Ko-Teilnehmer*innen der Untersuchung geschaffen. Wahrscheinlich ist, dass sich die Teilnehmenden hierdurch nicht allein zur 
Erläuterung, sondern immer wieder auch zur abwehrenden Rechtfertigung ihrer Ansichten, Verhaltensweisen und sozialen Beziehungen genötigt gesehen haben und der Bedarf an Abwehrhandlungen gewissermaßen also erst im Kontext der Gruppendiskussion und des Interviews entstanden ist. Aus diesem Grunde lässt die Untersuchung keine generalisierenden Feststellungen über das Verhalten der Teilnehmenden in natürlichen sozialen Kontexten $\mathrm{zu}$.

\subsection{Empirische, theoretische und praktische Anschlussfragen}

Verschiedene Fragen, die sich in der Auseinandersetzung mit dem untersuchten Material, der Reflexion seiner Ergebnisse sowie im Anschluss an die Befunde ergeben, müssen an dieser Stelle unbeantwortet bleiben. Hierbei handelt es sich m.E. insbesondere um folgende:

a) Wie weit reicht die Geltung der Befunde und wie relevant sind die theoretischen Ergebnisse?

b) Sind die in der vorliegenden Arbeit wiederholt investierten Hypothesen zu 'zweierlei Lernprozessen und einer >Normalisierung von Abwehr <riftig?

c) Wie kann abwehrendem Verhalten praktisch begegnet werden?

Einige Hinweise, wie diese beantwortet werden können, sollen allerdings im Folgenden präsentiert werden.

\section{Ad 1) Erweiterung der Geltungsreichweite der Ergebnisse und weiterer Forschungsbedarf}

Da die Geltungsreichweite der in dieser Untersuchung gewonnenen Ergebnisse unklar bleibt, wäre eine Untersuchung von größeren Stichproben an deutschen Universitäten lohnenswert. Hierfür bietet sich eine quantitative Erhebung an, um eine Schätzung zur Verbreitung von antisemitismusbezogenen Abwehrhaltungen vorzunehmen. Denkbar wäre hierbei z.B. die Durchführung eines faktoriellen Surveys (Vignettenanalyse), bei dem Reaktionsmuster auf Situationsbeschreibungen systematisch erhoben werden (vgl. Jasso/Opp 1997). Der Stimulus einer solchen Untersuchung wäre hierbei deutlich kürzer zu fassen als derjenige, der in der vorliegenden Studie verwendet wur- 
de. ${ }^{10}$ Denkbar wäre z.B. Studierenden Ergebnisse der quantitativen Antisemitismusforschung vorzulegen. Die in der vorliegenden Fallstudie generierten Beschreibungen zu den verschiedenen Formen von Abwehrargumentationen könnten hierbei für die Formulierung von Fragebogenitems genutzt werden. Zudem könnten Korrelationsanalysen zu den Zusammenhängen zwischen verschiedenen Abwehrargumentationen vorgenommen werden. So ließe sich dann z.B. fragen, ob Untersuchungsteilnehmer*innen, die die Erinnerung des Judenmords abwehren, auch eher dazu geneigt sind auf die Thematisierung von aktuellen Formen von Antisemitismus mit Abwehrargumenten $\mathrm{zu}$ reagieren. Eine besondere Herausforderung bestünde hierbei zweifellos darin, ein Forschungsinstrument zu entwickeln, mit dem die hier gewonnenen Hypothesen zu Motiven der Abwehr überprüft werden könnte. Hierfür müsste z.B. gezeigt werden, dass sich mithilfe von Variablen die nationalistische, antisemitische, israelfeindliche Einstellung messen, eine Neigung zur Verwendung von Abwehrargumentationen kausal erklären lässt (vgl. für einen entsprechenden Versuch Bergmann/Erb 1991b).

Eine weitere Möglichkeit die Relevanz der erhobenen Befunde aufzuzeigen, liegt in der Übertragung der theoretischen Befunde in andere Forschungsfelder. Alternativ zu Diskursanalysen (vgl. Knappertsbusch/Höttemann 2019) oder zu dem hier präsentierten, methodenintegrativen Forschungssetting wäre es insbesondere sinnvoll, ethnographische Studien durchzuführen, um Abwehrhandlungen in ihrem natürlichen Kontext zu untersuchen. Denn auch wenn in der vorliegenden Studie versucht wurde, sich durch ein alltagsnahes Untersuchungssetting an entsprechenden Situationen anzunähern, so ist festzuhalten, dass die Gruppendiskussionen und Interviews eine vergleichsweise künstliche Untersuchungssituation darstellten. Dementsprechend könnten etwa hinsichtlich der Verbreitung

10 Diese Empfehlung gilt auch für weitere qualitative Untersuchungen. Mit insgesamt 851 Wörtern war der Stimulus der vorliegenden Studie in etwa genau so lang wie der beim vom Frankfurter Institut für Sozialforschung durchgeführten Gruppenexperiment (732 Wörter). Allerdings wurde in ihm eine wesentlich größere Breite an möglichen Themen gesetzt. In der Analyse der Reaktionsmuster führte die Komplexität deshalb immer wieder dazu, dass unklar war auf welche Bestandteile des Reizes sich die Teilnehmer*innen in ihren Äußerungen bezogen. Thesen hierüber anzustellen ist allerdings - wie auch in Mertons Studien mit fokussierte Gruppendiskussionen (vgl. Merton/Kendall 1993) - unerlässlich, da hierdurch z.B. Thesen darüber aufgestellt werden können, welche Bestandteile einer Informationsquelle Teilnehmer*innen in besonderem Maße irritieren. 
von Antisemitismusbezogenen Abwehrargumentationen internationale Vergleiche aufgestellt, andere Milieus oder politische und zivilgesellschaftliche Institutionen untersucht werden und hierbei in Anschluss an Robert Beyer zwischen »Interaktionsöffentlichkeit«, »Themen-« oder »Versammlungsöffentlichkeit« (vgl. Beyer 2008: 45f.) unterschieden und überprüft werden, ob Abwehrhandlungen, auf dieser spezifischen Ebene, auf unterschiedliche Weise realisiert werden. Wenn z.B. Ionescu und Salzborn feststellen, dass der innerparteiliche Umgang mit Antisemitismus in allen Parteien häufig von Vermeidungsverhalten geprägt ist (vgl. Ionescu/Salzborn 2014) - ein Befund der von David Hirsh in einer aktuellen Studie auch in Hinsicht auf den Umgang der Labourparty des Vereinigten Königsreichs, insbesondere mit israelbezogenen Antisemitismus, bestätigt wird (Hirsh 2018: 66ff.) -, so lassen sich mit Hilfe eines Konzepts der Abwehr nicht nur die vielfältigen »Entsorgungsstrategien« von Antisemitismus auf den verschieden Interaktionsebenen kategorisieren, sondern hypothesengeleitet auch die möglichen Funktionen dieses Verhaltens untersuchen. ${ }^{11}$

Auch wäre interessant sich mit dem Konzept der Abwehr an aktuelle Diskusentwicklungen anzunähern. Diesbezüglich lässt sich feststellen, dass öffentliche Diskurse über (israelbezogenen) und insbesondere migrantischen Antisemitismus aktuell so rege geführt werden wie seit Jahren nicht mehr. Die Faktoren, die dies erklären, sind hierbei vielfältig. Offensichtliche $\mathrm{Zu}$ sammenhänge bestehen zwischen einer Zunahme antisemitischer Übergriffe und der Berichterstattung hierüber, einer gewachsenen Wahrnehmung der von islamistischem Terror ausgehenden Gefahren, aber auch der Bedeutungszunahme rassistischer Diskurse im Zuge der sogenannten $>$ Flüchtlingskrise $\lessdot$ Dies hat unterschiedliche Auswirkungen. Zum einen wird vermehrt über Antisemitismus gesprochen, wobei auch diejenigen zu Wort kommen können, die Antisemitismus als unteilbares Problem und als integralen Bestandteil der deutschen Gesellschaft betrachten. Zum anderen wird insbesondere im Rechtspopulismus versucht, über die Problematisierung von Antisemitismus vermeintlich Fremde zu stigmatisieren. Antisemitismusvorwürfe werden hierbei in strategischer Absicht verwendet. Vor diesem Hintergrund wä-

11 So kann z.B. gefragt werden, ob Mitglieder der Parteien hierdurch versuchen, die Beziehungen der Partei zu anderen politischen Akteuren zu stabilisieren, ihre Aktionen auf eine Stabilisierung des Außenbildes der Partei zielen oder es parteiinterne Solidarisierungszwänge gibt, so dass durch die Abwehr auch interne soziale Kohärenz hergestellt wird. 
ren Folgestudien wünschenswert, die die Frage behandeln, ob die wachsende Zunahme dieser Diskurse auch zu einer Sensibilisierung von Teilen der Bevölkerung über Antisemitismus als Gegenwartsphänomen führt und inwiefern und unter welchen Bedingungen diese Sensibilisierung wiederum durch die Externalisierung und damit durch Abwehrverhalten geprägt wird.

Des Weiteren wäre es sinnvoll, das Konzept der Abwehr als soziale Handlung auf andere Diskurszusammenhänge zu übertragen und hiermit auf seine interne Konsistenz hin zu überprüfen. Denkbare Themenbereiche wären z.B. der Umgang mit anderen Phänomenen der Menschenfeindlichkeit, des (antimuslimischen) Rassismus, Sexismus, der Homophobie usw. Hier kann die im Rahmen der vorliegenden Arbeit entwickelte Systematik von Abwehrhandlungen genutzt und gefragt werden, ob diese noch weiter ausdifferenziert werden muss. Insbesondere lohnenswert ist die Beantwortung der Frage, welche (situations- und themenspezifischen) Faktoren dazu führen, dass eine bestimmte und keine andere Abwehrhandlung gewählt wird. Diese Frage konnte in der vorliegenden Untersuchung immer nur gestreift werden. Damit eröffnet sich ein weites Kooperationsfeld, insbesondere für die Rassismusund Antisemitismus- sowie die Genderforschung.

\section{Ad 2) Überprüfung investierter Vorannahmen}

Für eine Erhärtung vieler der in dieser Arbeit vorgenommenen Deutungen ist es weiterhin notwendig, zwei in die Deutungen investierte Hypothesen zu überprüfen. So wurde in der Auseinandersetzung mit dem Theorem der Kommunikationslatenz argumentiert, dass unter Latenz Formen der Thematisierung des Antisemitismus bzw. antisemitischer Ereignisse gefasst werden können, die entweder auf die Abdrängung von antisemitischer Kommunikation oder von Antisemitismuskritik zielen. Wenn diese beiden Phänomene unter den Begriff der Latenz subsumiert werden können, folgt daraus, dass vom Vorliegen zweier gesellschaftlicher Lernprozesse ausgegangen werden muss, die gleichermaßen als Bestandteile der bundesdeutschen Kultur zu betrachten sind. Deshalb sind Folgestudien wünschenswert, die überprüfen, ob diese Annahme zutreffend ist und falls dem so ist, die Entwicklung beider Phänomene historisch vergleichend rekonstruieren. Hierbei kann sich als produktiv erweisen, das Wechselspiel historisch-spezifischer Thematisierungs- und Kritikformen des Antisemitismus auf der einen und Abwehrhandlungen auf der anderen Seite in den Fokus der Analyse zu rücken. 
Damit zusammenhängend wurde im Theoriekapitel die Überlegung aufgestellt, dass die Normalisierung bestimmter Abwehrargumentationen zu einer Verbreitung gesellschaftlicher Deutungsmuster und Konsensstrukturen führen kann, die es erschweren, das Problem Antisemitismus nachhaltig bekämpfen zu können. In Kapitel 2 wurde argumentiert, dass alle Formen von Abwehrargumentationen geeignet sind, solche Wirkungen zu entfalten. Allerdings fehlen empirische Nachweise über diese Prozesse und ihre Relevanz als Erklärungsansatz. Deshalb sind entsprechende historische Vergleichsstudien mit einer der vorliegenden Studie ähnlichen theoretischen Rahmung wünschenswert.

\section{Ad 3) Wie abwehrendem Verhalten begegnen?}

Wie bereits in der Einleitung deutlich gemacht wurde, stellt abwehrendes Verhalten eine große Herausforderung für die antisemitismuskritische Praxis dar, insofern es deren Bemühung, Antisemitismus sichtbar zu machen und antisemitisches Verhalten zu sanktionieren, effektiv unterläuft. Im Rahmen der abschließenden Diskussion der Ergebnisse kann hier kein Patentrezept zu einem effektiven Umgang mit entsprechenden Handlungen, die sich ja gerade durch ihre Vielfältigkeit auszeichnen, gegeben werden. Allerdings sollen an dieser Stelle einige Überlegungen zumindest kurz skizziert werden.

Abwehr ist nicht nur ein analytisch verwendbarer Begriff, sondern auch ein Reizwort. Wer es benutzt, geht - mit besseren oder schlechteren Argumenten - davon aus, dass eine Person oder Personengruppe einen offen geführten Diskurs aus bewussten oder unbewussten Motivlagen heraus vermeidet. Zweifellos können entsprechende Behauptungen zu Kränkungen führen und wiederum Abwehr provozieren. In der vorliegenden Studie wurde versucht, Thesen über Motive der Abwehr von Thematisierungen und Kritik des Antisemitismus mithilfe einer extensiven Feinanalyse des vorliegenden Materials zu entwickeln und hierbei die individuellen Kontexte von Sprechhandlungen zu berücksichtigen. Entsprechende Analysen sind äußerst arbeitsaufwendig und setzen die "Entlastung vom Handlungsdruck « voraus (Reichertz 2016: 260). Dementsprechend wurde zeitlich ausschweifende Arbeit in die Analyse der Daten investiert. In diesem Zusammenhang wurden Hypothesen aufgestellt und wieder revidiert. Demgegenüber müssen in der politischen Praxis ad-hoc Lösungen für aktuelle Probleme und Äußerungen gefunden werden, deren Ursachen oftmals in weiten Teilen im Dunkeln liegen. Damit steigt die Gefahr von Missinterpretation deutlich. Diese Problematik wird noch da- 
durch verschärft, dass nicht jede Handlung, die wie eine Abwehrhandlung anmutet, auch eine mit latentem Sinn versehene Abwehrhandlung sein muss. Daraus lässt sich der Schluss ziehen, dass es für die politische Praxis risikoloser und in bestimmten Situationen auch erfolgsversprechender sein dürfte, negative Reaktionen auf Antisemitismuskritik nicht vorschnell unter den Begriff der Abwehr zu subsumieren bzw. auf israelfeindliche und antisemitische Einstellungen zurückzuführen. Als alternativer Begriff für die Praxis kann demgegenüber z.B. der Begriff der >Dethematisierung von Antisemitismus dienen. Auch mit diesem lassen sich entsprechende Handlungen kritisieren. In diesem Falle wird vor allem normative Kritik geübt, die auf den problematischen Effekt entsprechender Handlungen - dem Verschwinden von Antisemitismus als Problem - abhebt. Die Kritik entsprechender (Sprach)Handlungen könnte hierdurch für manche Menschen auch annehmbarer gemacht werden. Auf der anderen Seite sollte festgehalten werden, dass die Deutung einer Handlung als Abwehrhandlung immer auch wohlmeinend zum Anlass genommen werden kann, latente Handlungsmotive zu reflektieren und das eigene Handeln zu ändern.

Weiterhin basieren Abwehrhandlungen in Reaktion auf anti-antisemitische Interventionen u.a. auf verzerrten und verzeichnenden Darstellungen sowohl der Kritiker*innen von Antisemitismus als auch antisemitischer Kommunikation. Für aufmerksame Diskursteilnehmer"innen ergibt sich hieraus die Möglichkeit zu intervenieren, noch bevor sich entsprechende Darstellungen in Gesprächen oder Diskursen etablieren können. Wie dies in einigen Situationen praktisch gelingen kann, wurde in Kapitel 5.11 gezeigt. Falschen Generealisierungen kann hierbei beispielsweise durch differenzierende Hinweise begegnet werden. Allerdings ist festzuhalten, dass entsprechende Interventionen durchaus anspruchsvoll sind, da abwehrende Argumentationen häufig auf beiläufige und unauffällige Weise formuliert werden.

Wichtig ist, der Institutionalisierung von Deutungsmustern, die die Dethematisierung von Antisemitismus befördern, entgegenzutreten. In Anschluss an Anja Weiß ist hierbei davon auszugehen, dass die Abwehr der Thematisierung und Kritik von Antisemitismus nicht nur auf mögliche Mängel anti-antisemitischer Strategien, sondern auch auf die »Stabilität dominanzkultureller [...] Klassifikationen" (Weiß 2001: 84) verweist. Das heißt, solange es in bestimmten Gruppen als selbstverständlich gelten kann, dass Antisemitismus allein als Problem der nationalsozialistischen Vergangenheit, als Form der Vernichtung und extremen Abwertung von Jüd*innen, als ein Problem von Randgruppen und »Anderen« zu verstehen ist, und 
heute vor allem Antisemitismusvorwürfe und nicht Antisemitismus das wesentliche Problem darstellten, wird es aus objektiven Gründen schwierig sein, Individuen, die diese Überzeugungen teilen, von der Gegenwart des (israelbezogenen) Antisemitismus zu überzeugen. Diejenigen, die die Gegenwart des Antisemitismus in seinen unterschiedlichen Formen ernst nehmen, müssen sich deshalb - wie es auch vielerorts in mühevoller (bildungs-)politischer Arbeit geschieht - entsprechenden Klassifikationen, Deutungs- und Konsensstrukturen rezeptionsfähige Alternativen entgegensetzen.

\subsection{Schluss}

Die gesellschaftspolitische Relevanz des israelbezogenen Antisemitismus hat seit Beginn der Erhebung der Daten für die vorliegende Studie nicht ab- sondern zugenommen. So stimmten einer repräsentativen Studie der Friedrich-Ebert-Stiftung zufolge im Jahr 2016 40\% der Antwort gebenden Teilnehmer*innen der Aussage zu, dass sie aufgrund der israelischen Politik "gut verstehen« könnten, dass man »etwas gegen Juden« habe (vgl. Zick et al. 2016: 43). Werden demgegenüber Jüd*innen gefragt, wie oft sie in den letzten 12 Monaten für die Politik des Staates Israel verantwortlich gemacht worden seien, so bejahen dies fast $80 \%$ der Teilnehmer*innen einer Studie des Jahres 2017 (vgl. Zick/Hövermann et al. 2017: 19). Demgegenüber verweist eine neue Studie zu Antisemitismus im Internet auf die signifikante Zunahme antisemitischer Kommentare in Kommentarspalten und sozialen Medien (vgl. Schwarz-Friesel 2018: 6).

Die (Fort-)Existenz von Antisemitismus ist also zweifelsohne ein gesellschaftliches Problem. Gedeihen kann Antisemitismus allerdings nur in einem Umfeld, in dem kein Widerspruch geäußert wird. Für eine antisemitismuskritische Kultur bedarf es hierbei nicht allein einem abstrakten Problembewusstsein dafür, dass Antisemitismus nicht sein sollte - dieser Konsens existiert nach wie vor - es bedarf zuallererst einer Sensibilisierung für seine aktuellen Ausdrucksformen, dafür, dass er als »bewegliches Vorurteil« (Adorno et al. 1969: 214) in allen gesellschaftlichen Schichten und Gruppen zu finden ist und deshalb auch von ideologisch sunverdächtigen oder politisch oder sozial nahestehenden Personen artikuliert wird. Die Existenz von den dem Abwehrphänomenen zugrundeliegenden vielfältigen Motivlagen steht dieser Auseinandersetzung entgegen. Denn Abwehr normalisiert Antisemitismus, insbesondere im Kontext alläglicher Interaktionen. 
Relevanz hat das Konzept der Abwehr als soziale Handlung allerdings nicht nur für aktuelle antisemitismusbezogene Diskurse. Eine Sensibilisierung für die Vielgestaltigkeit solcher Handlungen führen zu ihrer Sichtbarmachung als omnipräsentes Phänomen in aktuellen öffentlichen Debatten zu gesellschaftlichen Reizthemen. Das bedeutet, dass sich viele der Verhaltensmuster, die heute als Krise oder Zerfall öffentlicher Diskursnormen diskutiert werden, als Ausdruck einer Konfliktsituation beschreiben lassen, in der die beteiligten Konfliktakteur*innen nicht Kritik prüfend, wahrhaftig, faktenbasiert und mit Argumenten, sondern nur mit Abwehr reagieren und öffentliche Diskurse dadurch systematisch verzerren. Insbesondere die Handlung der moralischen Diskreditierung von Kritik, die nicht selten mit einer allgemeinen Disqualifizierung der Medien (`Lügenpresse`, >Fakenews`) einhergeht, ist zur Standardhandlung populistischer Akteure wie Pegida oder Donald Trump avanciert. Sie geht aber mit anderen sozialen Abwehrhandlungen Hand in Hand. Die vermeintlichen widersprüchlichen Positionierungen, die hieraus resultieren, wären nicht psychologisch, sondern nur sozial verstehbar. Genutzt wird immer das, was gerade Wirkung verspricht.

Es zeigt sich also, dass die Analyse der Abwehr von möglicher oder wahrscheinlicher Kritik anti-antisemitischer Interventionen nicht - wie die Antisemitismusforschung im Allgemeinen (vgl. z.B. Holz 2004) - einer Abgrenzung zu anderen Formen der Menschenfeindlichkeit auf der Ebene ihrer Gegenstandsbestimmung bedarf, sondern als spezialisierter Fall einer allgemeineren soziologischen Analyse zu verstehen ist. Aus diesem Grund kann sie von den Ergebnissen anderer Forschungsbereiche profitieren, wie umgekehrt diese von ihr. Die soziologische Analyse von Abwehrverhalten könnte sich damit zugleich auch als wichtiger Bestandteil soziologischer Gegenwartsdiagnostiken erweisen.

In der Einleitung wurde darauf hingewiesen, dass die Analyse des Umgangs von Student*innen mit dem Thema Antisemitismus unter anderem deshalb interessant ist, weil es sich hierbei um genau diese Gruppe handelt, aus der sich die demokratischen Eliten der Zukunft rekrutieren. Studienabgänger*innen besetzen häufig wichtige Positionen in Betrieben, staatlichen, politischen und anderen zivilgesellschaftlichen Organisationen und wirken dort als Multiplikator*innen gesellschaftlichen Wissens und als Vorbilder. Wenn die Annahme zutrifft, dass das für die vorliegende Studie zusammengestellte Sample zwar keinen repräsentativen, aber einen informativen Einblick zum Umgang mit Antisemitismus an einer deutschen Universität ermöglicht, so ist nun allerdings festzustellen, dass in diesen gesellschaftli- 
chen Schlüsselfeldern (weiterhin) Verhaltensweisen verbreitet sein werden, die Antisemitismus diskursiv als Problem entsorgen. Von einzelnen Teilnehmer*innen abgesehen handelt es sich hierbei nicht um radikale Extremfälle, auch wenn sie bisweilen antisemitische Positionen beziehen oder entsprechende Stereotype reproduzieren.

Insgesamt ist bemerkenswert, dass innerhalb der untersuchten studentischen Gruppe die Solidarisierung mit Grass und die Zurückweisung der Meinung der Kommentator*innen, die im Stimulusmaterial zur Sprache kamen, meist reibungslos erfolgte. Die Zurückweisung anti-antisemitischer Interventionen wirkte hierbei auf die nicht-jüdischen, deutschen Teilnehmer"innen, seien sie - wie in Kapitel 6 ausführlich erläutert - politisch orientiert oder desorientiert, nationalistisch eingestellt oder nicht, links, liberal oder konservativ - in hohem Maße integrierend. Ähnlich wie beim symbolischen Rassismus als Leugnung gegenwärtiger Diskriminierung oder Antifeminismus können solche Solidarisierungsprozesse demnach als ein "symbolic glue« (Kováts et al. 2016) verstanden werden, der eine gemeinsame Identität stiftet und Differenzen überdeckt. 
